\title{
Grass pollen immunotherapy for 3 to 4 years was still effective 3 years after being discontinued
}

Durham SR, Walker SM, Varga E-M, et al. Long-term clinical efficacy of grass-pollen immunotherapy. N Engl J Med 1999 Aug 12;341:468-75.

\section{QUESTION: In patients with grass pollen allergy successfully treated with immunotherapy for 3 to 4 years, does discontinuing maintenance immunotherapy result in increased symptoms and use of rescue medication?}

\section{Design}

A 3 year randomised \{allocation concealed*\}, † blinded (patients and investigators), ${ }^{*}$ placebo controlled trial.

\section{Setting}

A hospital allergy clinic in London, United Kingdom.

\section{Patients}

32 patients (59\% men) who had a history of severe seasonal allergic rhinitis, poor control of symptoms, and a positive skin prick test to timothy grass pollen extract and who had completed 3 years of maintenance therapy with grass pollen injections. Exclusion criteria were history of other allergies or medical illnesses and chronic asthma; patients with mild asthma were included if their symptoms were controlled by inhaled sympathomimetic $\beta_{2}$ adrenergic agonist bronchodilators. 15 matched, but not randomised, patients with allergic rhinitis who had never received immunotherapy were recruited as a control group. Follow up was $84 \%$.

\section{Intervention}

16 patients were allocated to continued monthly subcutaneous injection immunotherapy with a standardised, aluminium hydroxide adsorbed, depot grass pollen vaccine ( $1 \mathrm{ml}$ injection contained 100000 subcutaneous units, equivalent to 10000 biological units and containing $20 \mu \mathrm{g}$ of the phleum allergen P5). 16 patients were allocated to placebo injections containing aluminium hydroxide and histamine, $0.01 \mathrm{mg} / \mathrm{ml}$. For 3 years, $1 \mathrm{ml}$ injections were given monthly, except during pollen seasons, when the maintenance dose was reduced by $40 \%$.

\section{Main outcome measures}

Patient symptoms (total weekly scores of individual symptoms and a visual analogue score) and need for rescue medications were recorded daily in patient diaries from May through September each year.

Sources of funding: Medical Research Council; National Asthma Campaign (UK); ALK Abelló.

For correspondence: Dr S R Durham, Department of Upper

Respiratory Medicine, Imperial College School of Medicine, National Heart and Lung Institute, Doverhouse Street, London SW3 6LY, UK. Fax +441713518949.

Main results

Total symptom and rescue medication scores during the 11 week peak pollen season and visual analogue scores for a 1 week period during peak pollen season were reported as areas under the curve. During 1993, 1994, and 1995, the continued immunotherapy and placebo groups did not differ for total symptom scores $(\mathrm{p}=0.85$, 0.53 , and 0.60 , respectively), visual analogue scores $(\mathrm{p}=0.13,0.92$, and 0.87 , respectively), or need for rescue medication $(\mathrm{p}=0.85,0.96$, and 0.88 , respectively). The 15 matched control patients continued to have seasonal symptoms.

\section{Conclusion}

Grass pollen immunotherapy for 3 to 4 years continued to reduce symptoms and the need for rescue medication for 3 years after discontinuing immunotherapy injections.

*See glossary.

$\dagger$ Information provided by author.

\section{COMMENTARY}

Several well controlled clinical trials show that allergen immunotherapy is effective in the treatment of allergic rhinitis. ${ }^{1}$ This study by Durham and colleagues shows that the beneficial effects of immunotherapy in allergic rhinitis persist for as long as 3 years after immunotherapy is discontinued. The study was double blind, placebo controlled, and the longest of its kind. Furthermore, objective measures of hypersensitivity (cutaneous late phase response to allergen challenge and infiltration of cells containing interleukin- 4 mRNA) confirmed the prolonged benefits of immunotherapy.

Clinicians can now be more confident in recommending allergen immunotherapy for patients with allergic rhinitis. Not only may patients experience control of symptoms while taking allergen immunotherapy injections, but the immunotherapy may also modify the long term course of the disease.

Because immunotherapy for rhinitis should only be considered in patients with a clear diagnosis of allergy, clinicians now have more reason to make a specific diagnosis of allergic rhinitis in patients who present with upper respiratory symptoms. Workup must include a careful medical history combined with the results of immediate hypersensitivity testing (allergy skin testing or in vitro measurement of specific IgE antibody).

The results of this study also provide guidance for determining the duration of immunotherapy in allergic rhinitis. For many patients, 3 or 4 years of immunotherapy may control symptoms for years, even after immunotherapy is discontinued. Physicians and patients considering immunotherapy for allergic rhinitis no longer have to worry that initiating treatment commits the patient to a lifetime of injections.

For each patient with allergic rhinitis, the physician must balance the benefits and risks of each treatment method. The results of this and similar studies show that a 3 year course of allergen immunotherapy (with the potential for long term benefits) may be more attractive than pharmacotherapy for many patients.

\section{James T Li, MD, PhD Mayo Clinic} Rochester, Minnesota, USA

1 Allergen immunotherapy: therapeutic vaccines for allergic diseases. Allergy 1998;53(Suppl 44):1-42. 